\title{
Dynamic Quenching of Triplet Excitons in Single Conjugated- Polymer Chains
}

\author{
Jakob Schedlbauer, Ullrich Scherf, Jan Vogelsang, and John M. Lupton* \\ Cite This: J. Phys. Chem. Lett. 2020, 11, 5192-5198 \\ Read Online
}

ABSTRACT: By measuring the fluorescence photon statistics of single chains of a conjugated polymer, we determine the lifetime of the metastable dark state, the triplet exciton. The single molecule emits single photons one at a time, giving rise to photon antibunching. These photons appear bunched in time over longer time scales because of excursions to the triplet dark state. Remarkably, this triplet intermittency in the fluorescence is spontaneously suppressed over time scales of seconds, implying that either triplet formation is inhibited or that triplets are selectively quenched without the singlet fluorescence being affected. Such discrete switching in the strength of photon bunching is only seen in highly ordered and rigid chains of a ladder-type conjugated polymer. It does not occur in single dye molecules. We propose that trapped photogenerated charges on the chain selectively quench triplets but not singlets, presumably because the effective diffusion length of triplets is longer along the highly rigid ladder-type backbone.

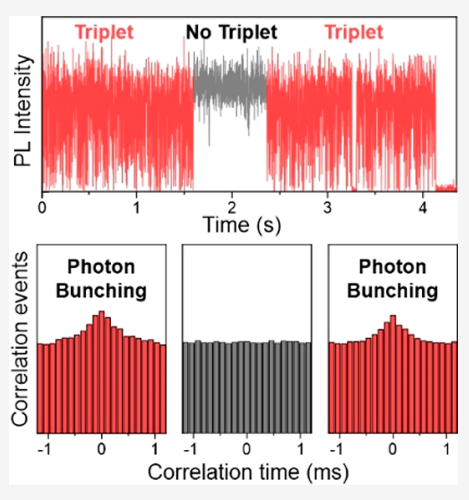

$\mathrm{T}$ he nature of the interaction between charges and singlet and triplet excitations of molecules is critical in many organic-electronic devices, particularly in organic light-emitting diodes (OLEDs). Both types of excitation may pass their energy to the charge in a Coulombic process not dissimilar to Auger recombination in semiconductors, ${ }^{1-5}$ lowering the binding energy of the charge to the molecule and potentially triggering chemical reactions. Whereas such nonradiative quenching of singlets is simple to study by considering the photoluminescence (PL) yield or PL lifetime, investigating the quenching of triplets requires either phosphorescence ${ }^{6}$ or photoinduced absorption measurements, ${ }^{7}$ or an inspection of delayed fluorescence arising from triplet-triplet annihilation. ${ }^{8}$ Magnetic resonance techniques can also be used, since the triplets are characterized by a distinct spin-1 resonance feature. ${ }^{9}$ However, detection of changes to the triplet population then typically occurs by a secondary observable, the change in PL from the singlet population, which in turn is modified due to the singlet-triplet quenching mechanism in ensemble measurements. Similarly, on the single-molecule level, magnetic resonance controls the lifetime of the triplet dark state and therefore the overall brightness of fluorescence. $^{10-13}$ A comparable indirect measurement of the dynamics of triplets is also offered by studying the photon statistics in the PL intensity on the level of single molecules as sketched in Figure 1a. Under photoexcitation, excursions to the triplet state will occur within the excited-state manifold, suppressing fluorescence and leading to pronounced intermittency. ${ }^{14-16}$ This suppression also arises for larger molecules with multiple chromophores if bichromophoric annihilation in the form of singlet-singlet annihilation ${ }^{17}$ (SSA) occurs efficiently between the emitting sites. In the rare event that two or more excitons are formed on the molecule during the singlet excited-state lifetime of $\sim 400$ ps, the excitation energy on different chromophores is funneled to one single chromophore by nonradiative energy transfer. Ultimately, only this one chromophore can decay radiatively, so that single-photon emission arises in the form of photon antibunching. The degree of antibunching is therefore a measure of the effectiveness of intramolecular energy transfer. ${ }^{17}$ If a triplet exciton is formed due to intersystem crossing (ISC), the emitting chromophore-and therefore the emission of the entire molecule-will be effectively turned off for the duration of the triplet lifetime. If multiple chromophores are present and these chromophores couple by dipole-dipole interactions, then the triplet state on one chromophore can absorb the excitation energy of adjacent chromophores, quenching the fluorescence by singlet-triplet annihilation (STA). ${ }^{17}$ Suppression of fluorescence can also occur by quenching of singlets by polarons, that is, singlet-polaron quenching (SPQ). ${ }^{2}$ Such quenching also arises in the triplet state (TPQ): ${ }^{2}$ the neutral excitation passes its excitation energy to the charge, raising it to a higher molecular orbital. ${ }^{12}$ Intermittent quenching of the fluorescence, blinking, can be resolved either directly in the time domain (Figure 1b),

Received: April 29, 2020

Accepted: June 8, 2020

Published: June 8, 2020 
a

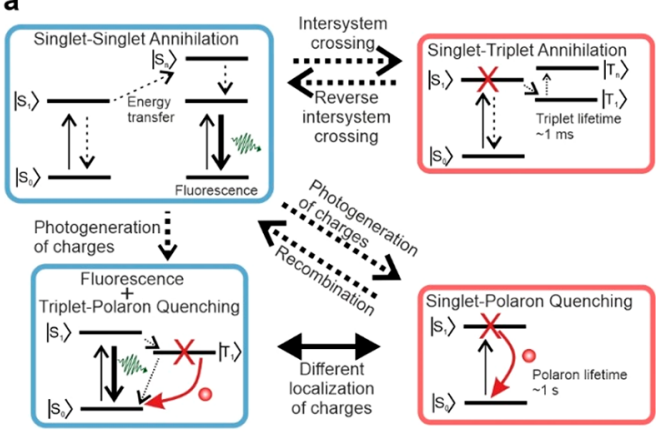

b

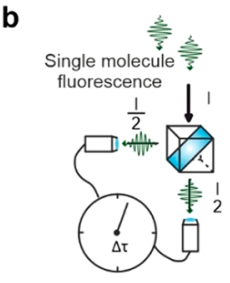

C
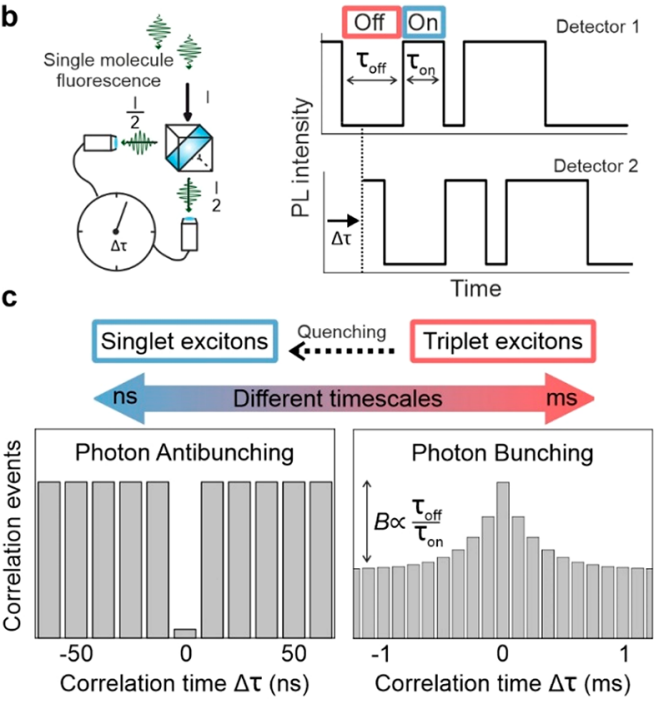

Figure 1. (a) Sketch of the interactions between different excitonic species in multichromophoric conjugated-polymer chains, i.e. SSA, STA, and quenching of singlet or triplet excitons by polarons (SPQ and TPQ). Blue boxes indicate on periods of the molecule, where it cycles between singlet ground and excited state, emitting photons. Red indicates off periods, where fluorescence is suppressed. The crosses indicate quenching of either the triplet or the singlet state. (b) Influence of dark states on the single-molecule PL intensity, measured in a Hanbury Brown-Twiss detection arrangement. Excursions into a nonemissive state give rise to strong fluorescence blinking quantified by characteristic on and off times of the fluorescence. (c) Sketch of photon correlation functions as photon coincidence histograms, on different time scales, visualizing the photon bunching and antibunching signatures of single-molecule fluorescence caused by STA and SSA, respectively. The photon bunching amplitude $B$ at $\Delta \tau$ $=0$ is proportional to the ratio between off and on times.

provided that enough photons can be detected, or in an intensity autocorrelation analysis (Figure 1c, left). ${ }^{18}$ Since a single chromophore emits precisely one single photon at a time, passing the fluorescence through a beam splitter and recording the coincidence rate on two photodetectors on either side of the beam splitter will yield a correlation amplitude of zero at zero delay time $\Delta \tau::^{19}$ photon antibunching occurs. Excursions from the singlet manifold of the molecule to the triplet excited state result in the opposite effect on longer time scales $(\sim 1 \mathrm{~ms})$ : single photons arrive in bursts, or bunches, because the molecule switches between an "on" state, where it cycles within the singlet manifold between the ground and the first excited state, emitting a continuous photon stream; and an "off" state, where the emission is quenched by triplets or polarons. The lifetimes of these two quenching species differ by over 3 orders of magnitude, making
STA and SPQ easy to distinguish in the photon correlation; on the short time scales discussed here, only STA is of relevance. Fluorescence blinking gives rise to photon bunching signatures in the photon correlation function (Figure 1c, right), which decays exponentially in amplitude with correlation delay time $\Delta \tau$. Investigating this photon bunching signal offers direct access to triplet-state dynamics, allowing the triplet generation and recombination rate to be investigated and hence the characteristic on and off times of the fluorescence of the molecule, $\tau_{\text {on }}$ and $\tau_{\text {off }}$ to be determined. ${ }^{20}$ This approach has previously been used to study the interactions of triplet states with molecular oxygen. ${ }^{21-23}$

A particularly interesting case of the interplay between photon bunching and antibunching with direct relevance to organic electronics is posed by conjugated polymers. In a conjugated-polymer chain, triplet excitons will migrate by Dexter transfer, whereas singlets can either delocalize coherently or move incoherently between chromophores by a Förster-type process. Triplets are known to act as nonradiative trap sites for singlets by the STA mechanism. ${ }^{24,25}$ Together with the large difference in the exciton lifetime of singlet and triplet species, the effective diffusion lengths of the two species can differ, ${ }^{26}$ which in turn may affect interactions with localized charge carriers on the polymer chain. Here, we report on the unusual relationship between photon bunching and antibunching in a highly ordered rigid para-phenylene ladder polymer, phenyl-substituted ladder poly(para-phenylene) (PhLPPP, structure shown in Figure 2a). Although the macromolecule behaves as one single quantum emitter, showing perfect photon antibunching, ${ }^{29}$ the degree of photon bunching varies strongly over time, implying that the STA channel intermittently becomes mute. Since there is no obvious reason why triplet formation should be suppressed, we conclude that triplets can be quenched effectively without the singlet population being affected.

We prepared single-molecule samples by dispersing PhLPPP in poly(methyl methacrylate) (PMMA). The density of diffraction-limited spots seen under the microscope scales directly with analyte concentration, implying that aggregation is negligible. Figure $2 \mathrm{~b}$ shows the photon correlation histogram measured from one single molecule. Discrete bars, spaced in time by the period of the excitation laser $(12.5 \mathrm{~ns})$, are seen. The central peak at zero delay time between the two photodetectors offers a simple metric for the quality of photon antibunching, which, in this example, is quantified by a ratio of central to lateral peak height $\left(\frac{N_{c}}{N_{1}}\right)=0.04$. This value is only just above the ratio expected for a single emitter based on the signal-to-background ratio of the experiment $\left(\frac{N_{c}}{N_{1}}\right)_{\text {expected }}=0.02 .^{27,28}$ On much longer correlation time scales, the opposite effect sets in as shown in Figure 2c: photon bunching occurs because of excursions to the long-lived triplet state. The signal decays exponentially with the decay time of the autocorrelation, $\tau_{\text {ac }}$ which together with the correlation amplitude $B$ offers a metric for the triplet lifetime expressed as $\tau_{\mathrm{T}}=\tau_{\mathrm{ac}} \cdot B .^{29}$ A single-exponential fit of the data in panel c (red lines) returns a triplet lifetime of $\tau_{\mathrm{T}}=0.5 \mathrm{~ms}$. The on time of the fluorescence can be calculated as $\tau_{\text {on }}=\tau_{\mathrm{ac}} \cdot\left(1+\frac{1}{B-1}\right)=1.5 \mathrm{~ms}^{29}$

Photon bunching shows up as an intermittency in the singlemolecule fluorescence intensity trace as a function of time ${ }^{20}$ 


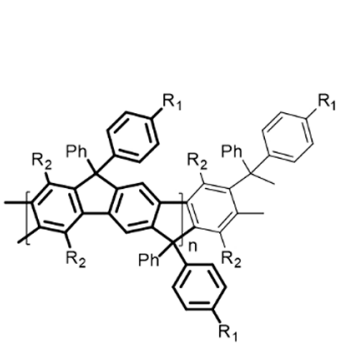

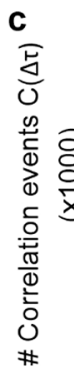

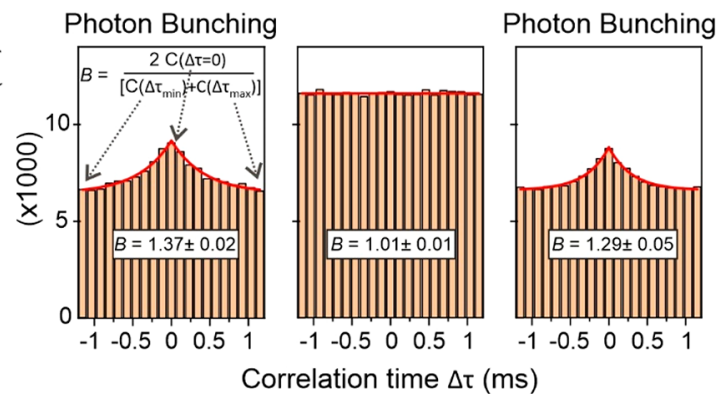

d

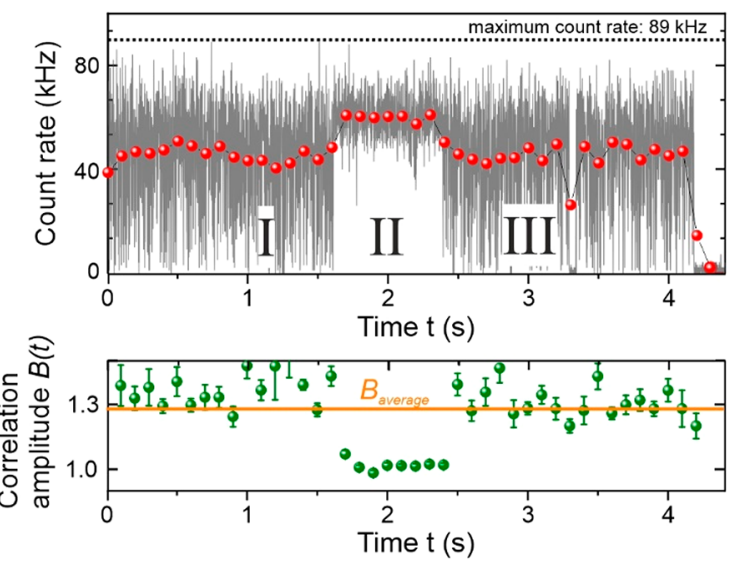

Figure 2. (a) Structure of the ladder-type poly(para-phenylene) (PhLPPP; $\mathrm{R}_{1}$ : decyl, $\mathrm{R}_{2}$ : hexyl). (b) Photon correlation histogram binned in intervals of the repetition period of the pulsed laser $(12.5$ ns). Because of the high brightness of single PhLPPP molecules, the correlation is measured for one single molecule, showing strong photon antibunching as quantified by the ratio $\left(\frac{N_{c}}{N_{1}}\right)=0.04$ of central to lateral coincidence peaks. Photon correlation histograms (c) calculated at three different times of the single-molecule photon stream shown in (d), together with offset single-exponential fits (red lines) used to extract the correlation amplitudes $B$. Correlation events were calculated up to a maximum correlation time of $\Delta \tau_{\max }=1.2 \mathrm{~ms}$. (d) Trace of the single-molecule PL intensity with a time binning of either $1 \mathrm{~ms}$ (gray line) or $100 \mathrm{~ms}$ (red dots), split into three regions (I, II, III). (e) Time evolution of the correlation amplitude $B(t)$ calculated for $100 \mathrm{~ms}$ time windows of the photon stream in (d). Error bars arise from the exponential fits to the correlation histograms. The orange line indicates the average correlation amplitude $B_{\text {average }}$.

(cf. Figure 1b), provided that the temporal binning of the detector photon count rate is sufficiently short, that is, on the time scale of the dark-state lifetime. Such a trace is shown in Figure $2 \mathrm{~d}$. The maximal fluorescence intensity remains constant over the measurement time of $4.2 \mathrm{~s}$, but, remarkably, two distinct regions of emission dynamics appear to exist: one region (II), where intermittency is minimal and the molecule persistently remains in the on state; and a second region (I, III) where the molecule switches on and off. Raising the integration window of photon counts from $1 \mathrm{~ms}$ (black line) to $100 \mathrm{~ms}$ (red dots) averages over multiple on-off cycles of the molecule, demonstrating that the time-integrated fluorescence intensity is indeed greater when the molecule does not undergo excursions to the dark state (II).

Computing the photon correlations for these distinct regions in Figure 2c shows that photon bunching only arises in periods I and III, indicating that the effect of the triplet excited state on the single-molecule fluorescence is suppressed for the time interval II. We can define the amplitude of photon bunching as $B=2 \cdot C(\Delta \tau=0) /\left[C\left(\Delta \tau_{\max }=1.2 \mathrm{~ms}\right)+C\left(\Delta \tau_{\min }=-1.2 \mathrm{~ms}\right)\right]$, that is, the ratio between the central bar $C(\Delta \tau=0)$ and the average of the outermost values of the correlation histogram, $C\left(\Delta \tau_{\max }\right)$ and $C\left(\Delta \tau_{\min }\right)$. To investigate changes in the photon correlation we calculate the time-resolved amplitude $B(t)$ for intervals of $100 \mathrm{~ms}$ in Figure 2e. In region (II), the correlation amplitude suddenly drops to unity, implying that the triplet state is no longer quenching the fluorescence of the molecule.

To investigate this phenomenon in more detail, we studied the distribution of the effective amplitude of photon bunching for a total of 1533 single chains. Figure 3a plots this
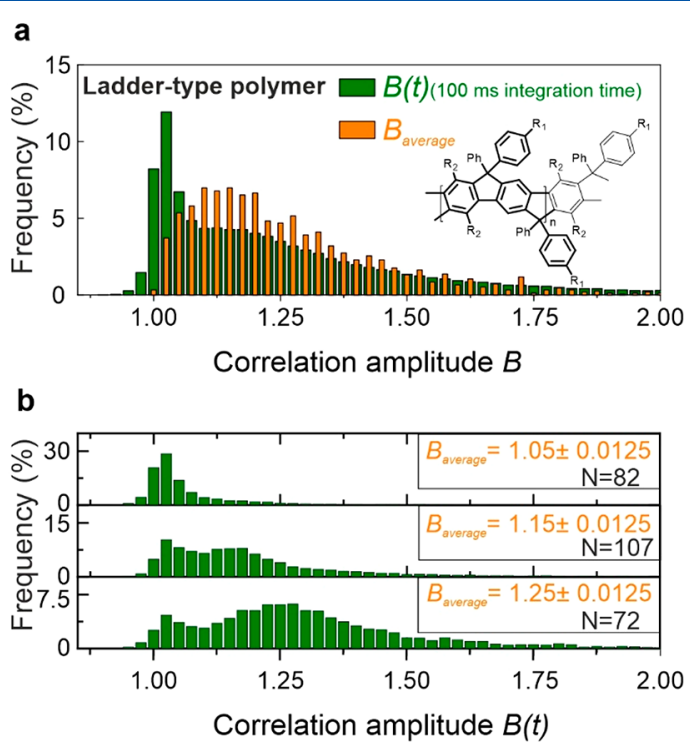

Figure 3. (a) Distributions of single-molecule correlation amplitudes $B_{\text {average }}$ as defined in Figure 2, measured for 1533 single PhLPPP molecules, computed for the entire fluorescence intensity trace (orange bars). The maximum correlation time used, i.e., the range covered in the correlation indicated in Figure 1c, was $\Delta \tau_{\max }=1.2 \mathrm{~ms}$. Reducing the integration time of the single-molecule PL intensity trace used to calculate each $B$ value to $100 \mathrm{~ms}$ (green bars), i.e., sampling only $100 \mathrm{~ms}$ of the fluorescence intensity trace at once when computing the correlation function, reveals a bimodal distribution with a distinct peak arising at $B \approx 1$. (b) Distribution of $B(t)$ values for single molecules within a range of different $B_{\text {average }}$ values, computed with $100 \mathrm{~ms}$ sampling times. The number of single molecules used in each data set is stated. A bimodal distribution is apparent for molecules with larger $B_{\text {average }}$.

distribution for values calculated from the entire fluorescence intensity trace, that is, the values of $B_{\text {average }}$ (orange). The histogram is broad, and only $1 \%$ of all molecules show an absence of bunching, that is, $B=1$, for the entirety of the trace, implying that the photon bunching phenomenon is universal to virtually all single chains. However, since short temporal excursions to region (II) arise in which bunching is suppressed 
(i.e., $B \approx 1$ ), it is instructive to compute $B(t)$ in time slices of $100 \mathrm{~ms}$ for the entire data set, plotted in green. Time slicing has a dramatic effect on the distribution of $B$ values with a strong peak emerging close to unity and a flattening of the residual distribution. To enhance the contrast between temporal regions in each fluorescence trace of distinct photon bunching (I) and the absence thereof (II), we select singlemolecule PL intensity traces, which show different average bunching values. This analysis in Figure $3 \mathrm{~b}$ allows a clear differentiation between regions without dark-state-induced photon bunching (II) and regions with small but finite correlation amplitudes (I). For molecules with high $B_{\text {average }}$ values the distribution of $B(t)$ becomes bimodal.

Performing an analogous sequential analysis of the fluorescence of 684 single dye molecules of 4,4-difluoro1,3,5,7,8-pentamethyl-4-bora-3a,4a-diaza-s-indacene (BODIPY) dispersed in PMMA in Figure 4a shows no such bimodal

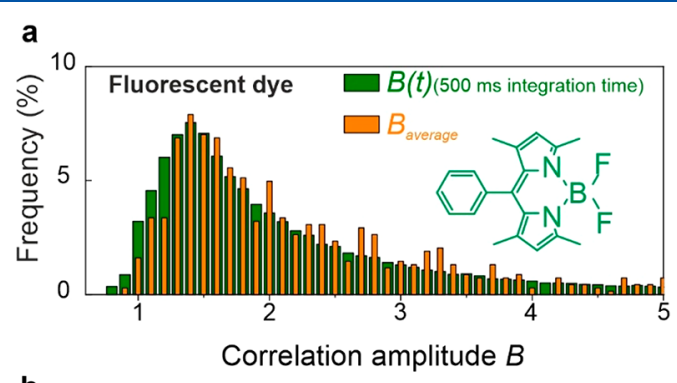

b

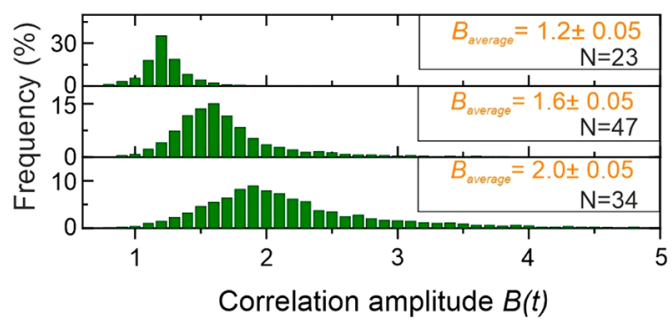

Figure 4. (a) Distributions of single-molecule correlation amplitudes $B_{\text {average }}$ for 684 BODIPY dyes (orange bars) with the structure of the dye inset. Correlation events were calculated up to a maximum correlation time of $\Delta \tau_{\max }=2.5 \mathrm{~ms}$. Reducing the integration time for calculating $B$ from the entire duration of the trace to $500 \mathrm{~ms}$ time windows (green bars) does not change the shape of the distribution, and, in contrast to Figure 3, no distinct peak is visible at $B \approx 1$. (b) Distribution of $B(t)$ values for single molecules within a range of different $B_{\text {average }}$ values, computed with $500 \mathrm{~ms}$ sampling times. The number of single molecules used in each data set is stated.

distribution, implying that the triplet state is continuously present as seen by its effect of quenching radiative singlet transitions. Even for a selection of single dyes with high $B_{\text {average }}$ values no bifurcation of the histogram is apparent in Figure $4 \mathrm{~b}$.

In principle, it is conceivable that the bimodal distribution of the time-dependent photon-bunching amplitude $B(t)$ in PhLPPP in Figure 3 arises from a suppression of triplet formation, but such an inhibition would necessitate a spontaneous and dramatic change in the effectiveness of ISC, which is implausible. Alternatively, the triplets may simply become quenched by a charge localized on or in the immediate vicinity of the molecule. ${ }^{1,2,6,12,30-33}$ In the case of PhLPPP, both SPQ and TPQ can occur. Clear signals of SPQ are apparent when the entire fluorescence switches off for several seconds, a universal feature of single-molecule spectroscopy at room temperature. ${ }^{30}$ Remarkably, region (II) in the fluorescence traces (cf. Figure 2d) appear to exist where only TPQ occurs but not SPQ. ${ }^{34}$ We propose that this difference arises from the fact that, in PhLPPP, the on-chain singlet diffusion length is shorter than the triplet diffusion length. ${ }^{35}$

In the single BODIPY dye molecule, if a charge forms, both singlet and triplet excitations are quenched, because there is only one single chromophore within the molecule. In BODIPY, the photon statistics of the fluorescence from the singlet state therefore always carries the photon-bunching signature of the triplet (i.e., $B(t)>1$, cf. Figure 4 ). This simple picture is not appropriate for most conjugated polymers, which can contain multiple chromophores: ${ }^{36,37}$ a charge need not quench the fluorescence of all chromophores. Crucially, poly(phenylene-vinylenes), ${ }^{38}$ polyfluorenes, ${ }^{39}$ poly(phenylene-butadiynylenes), ${ }^{40,41}$ and polythiophenes, ${ }^{25,42,43}$ which do exhibit both photon antibunching and photon bunching, signifying sufficient dipole-dipole coupling between chromophores for SSA to occur, do not show signatures of fluctuations of the amplitude of photon bunching, that is, of triplet lifetime. ${ }^{40}$ The present observation that TPQ can occur without SPQ appears to be unique to the highly ordered polymer backbone of PhLPPP. We propose that the exceptional degree of rigidity and the strong conjugation of the ladder-type backbone promote on-chain triplet diffusion by the Dexter mechanism, whereas the Förster transfer of singlets between different chromophores with inline dipoles in head-totail configuration is inherently less effective.

If TPQ occurs preferentially over SPQ in PhLPPP because of a greater effective diffusion length of triplets, it should be possible to determine an influence of chain length on the intermittency of the triplet state, that is, the apparent bifurcation of the photon bunching amplitudes $B(t)$. In short chains, in analogy to the BODIPY dye, charge formation should quench both singlets and triplets, so that the bifurcation of the $B(t)$ histogram is reduced. Figure 5 a illustrates the proposed quenching mechanism, where a photogenerated charge interacts with a mobile triplet but leaves the singlet population mostly intact. We use the overall "molecular brightness", ${ }^{20}$ that is, the fluorescence count rate, as a simple metric for the polymer chain length: the longer the chain, the larger the effective absorption cross section, the greater the overall maximum fluorescence rate. Following the above analysis, the probability for the photon-bunching signal to vanish for a given interval of the fluorescence intensity trace [region (II)] should increase with overall chain length and therefore brightness. We divide 1533 single PhLPPP molecules into three groups of low (b), intermediate (c), and high (d) intensity and plot the corresponding histograms of $B(t)$, computed in correlation time windows of $100 \mathrm{~ms}$. The number of molecules showing time intervals during which photon bunching is suppressed, that is, $B(t) \approx 1$, rises with increasing brightness. To quantify the average lifetime of the polarons responsible for SPQ we calculate the photon correlation amplitude of one single molecule over multiple orders of magnitude of the correlation time $\Delta \tau$ in Figure 5e (gray points). A biexponential functionality is apparent to the correlation curve, corresponding to intermittencies of the PL intensity due to STA and SPQ. A biexponential fit to the data (red line) yields the two characteristic off-times attributed to the triplet and polaron lifetimes. From an analogous correlation analysis for 100 single PhLPPP molecules we generate histograms of triplet and polaron lifetimes in Figure 

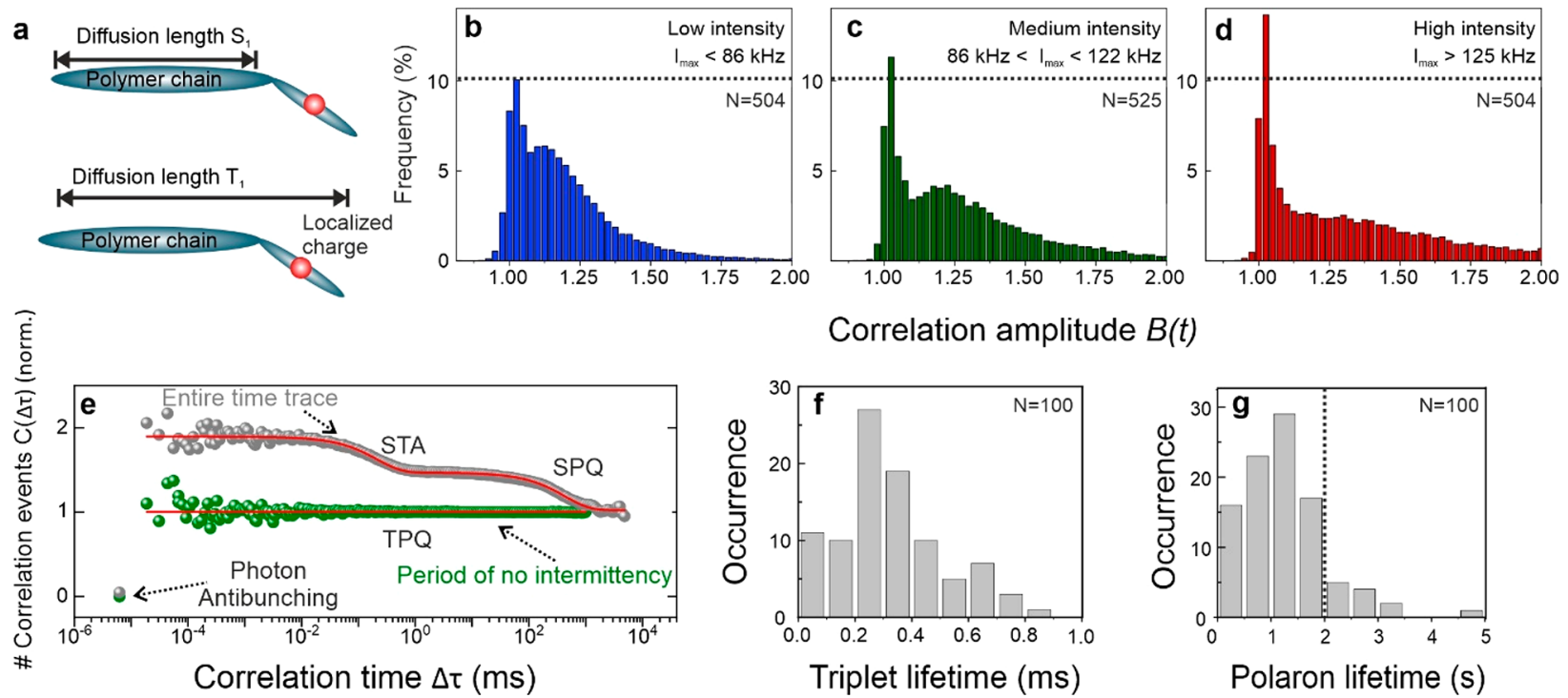

Correlation amplitude $B(t)$
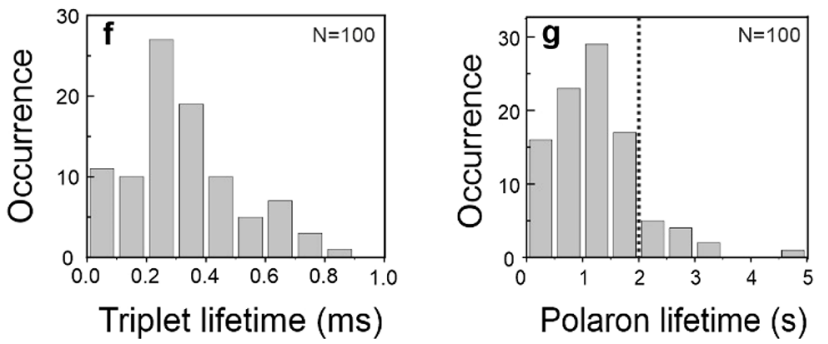

Figure 5. (a) Sketch of the proposed interplay between different effective on-chain diffusion lengths for singlet and triplet excitons and their possible interactions with photogenerated localized charges. $(\mathrm{b}-\mathrm{d})$ Distributions of the correlation amplitude values $B(t)$ calculated in $100 \mathrm{~ms}$ time windows of the PL intensity traces of 1533 single molecules split into groups of low, medium, and high emission intensities. The intensities $I_{\max }$ are stated in the plots. The dotted line is a guide to the eye. (e) Autocorrelation curve of an entire single-molecule time trace (gray dots). Green dots show the correlation function computed over a finite period where no intermittency occurs [i.e., region (II)]. We propose that this region arises due to TPQ. For comparison, both curves are normalized to saturate at a correlation value of $C(\Delta \tau \rightarrow \infty)=1$ for long correlation times. The two distinct photon-bunching amplitudes in the entire correlation correspond to PL quenching by triplets (STA) and, on longer time scales, polarons (SPQ). A biexponential fit to the data (red lines) allows the bunching amplitudes and decay times to be determined in order to calculate the characteristic off times in the fluorescence as determined by the triplet and polaron lifetimes. (f, $\mathrm{g}$ ) Distributions of triplet and polaron lifetimes for 100 single molecules. The dashed line in panel $(\mathrm{g})$ marks the maximum polaron lifetime of $\sim 2 \mathrm{~s}$, which can be extracted from the data with confidence (see Supporting Information).

5f,g. The median lifetimes of both species are determined as $\tau_{\mathrm{T}}$ $=0.31 \mathrm{~ms}$ and $\tau_{\mathrm{P}}=1.3 \mathrm{~s}$ for triplets and polarons, respectively. Calculating the correlation signal for an extended time period where no PL intermittency occurs [i.e., region (II)] removes the characteristic photon bunching signal so that the correlation curve is flat (green dots in Figure 5e). Since this time period is necessarily shorter than the entire time period over which fluorescence was recorded, the green dots do not extend as far out in $\Delta \tau$ as the gray dots do. Nevertheless, there is clearly no photon correlation apparent in the time region of SPQ in the green curve, implying that TPQ can occur without SPQ happening.

These findings are hard to rationalize with the suppression of photon bunching due to an inhibition of ISC in the excited singlet state, that is, a suppression of triplet formation. We conclude that triplet excited states must still be formed but become intermittently quenched, most likely by a photogenerated trapped charge or charge pair. ${ }^{2}$ Our proposed mechanism for selective quenching of triplet excitons illustrates the interplay between localization and on-chain mobilities of excitons on a single polymer chain. Extending this study of fluorescence photon statistics to mesoscopic molecular aggregates-where kinetics can additionally be rationalized in terms of interchain exciton diffusion-should open a route to probe three-dimensional charge and energy migration in conjugated-polymer systems. ${ }^{2}$ The results are relevant for strategies for managing triplets in devices. In OLEDs, triplet diffusion should be minimized to prevent TPQ and the associated loss due to omnipresent charge carriers, whereas in solar cells, it could be beneficial to exploit the TPQ mechanism so as to limit STA, which can constitute a major loss channel. ${ }^{20}$

\section{ASSOCIATED CONTENT}

\section{Supporting Information}

The Supporting Information is available free of charge at https://pubs.acs.org/doi/10.1021/acs.jpclett.0c01308.

Materials and methods for sample preparation and experimental setup, discussion of data analysis, additional references (PDF)

\section{AUTHOR INFORMATION}

\section{Corresponding Author}

John M. Lupton - Institut für Experimentelle und Angewandte Physik, Universität Regensburg, 93053 Regensburg, Germany; ○ orcid.org/0000-0002-7899-7598; Email: john.lupton@ ur.de

\section{Authors}

Jakob Schedlbauer - Institut für Experimentelle und Angewandte Physik, Universität Regensburg, 93053 Regensburg, Germany

Ullrich Scherf - Macromolecular Chemistry Group, Chemistry Department and Wuppertal Center for Smart Materials \& Systems CM@S, Bergische Universität Wuppertal, 42097 Wuppertal, Germany; 이이.org/0000-0001-8368-4919

Jan Vogelsang - Institut für Experimentelle und Angewandte Physik, Universität Regensburg, 93053 Regensburg, Germany; (1) orcid.org/0000-0001-7952-9342

Complete contact information is available at:

https://pubs.acs.org/10.1021/acs.jpclett.0c01308

\section{Notes}

The authors declare no competing financial interest. 


\section{ACKNOWLEDGMENTS}

Funded by the Deutsche Forschungsgemeinschaft (German Research Foundation), Project-ID 314695032-SFB 1277.

\section{REFERENCES}

(1) Hertel, D.; Meerholz, K. Triplet-polaron quenching in conjugated polymers. J. Phys. Chem. B 2007, 111 (42), 12075-12080.

(2) Gesquiere, A. J.; Park, S. J.; Barbara, P. F. Hole-induced quenching of triplet and singlet excitons in conjugated polymers. J. Am. Chem. Soc. 2005, 127 (26), 9556-9560.

(3) Coehoorn, R.; Bobbert, P. A.; van Eersel, H. Förster-type tripletpolaron quenching in disordered organic semiconductors. Phys. Rev. B: Condens. Matter Mater. Phys. 2017, 96 (18), 184203.

(4) Sahoo, D.; Tian, Y.; Sforazzini, G.; Anderson, H. L.; Scheblykin, I. G. Photo-induced fluorescence quenching in conjugated polymers dispersed in solid matrices at low concentration. J. Mater. Chem. C 2014, 2 (32), 6601-6608.

(5) Baker, W. J.; McCamey, D. R.; van Schooten, K. J.; Lupton, J. M.; Boehme, C. Differentiation between polaron-pair and tripletexciton polaron spin-dependent mechanisms in organic light-emitting diodes by coherent spin beating. Phys. Rev. B: Condens. Matter Mater. Phys. 2011, 84 (16), 165205.

(6) Kalinowski, J.; Stampor, W.; Mezyk, J.; Cocchi, M.; Virgili, D.; Fattori, V.; di Marco, P. Quenching effects in organic electrophosphorescence. Phys. Rev. B: Condens. Matter Mater. Phys. 2002, 66 (23), 235321.

(7) Osterbacka, R.; Wohlgenannt, M.; Chinn, D.; Vardeny, Z. V. Optical studies of triplet excitations in poly(p-phenylene vinylene). Phys. Rev. B: Condens. Matter Mater. Phys. 1999, 60 (16), 1125311256.

(8) Partee, J.; Frankevich, E. L.; Uhlhorn, B.; Shinar, J.; Ding, Y.; Barton, T. J. Delayed fluorescence and triplet-triplet annihilation in piconjugated polymers. Phys. Rev. Lett. 1999, 82 (18), 3673-3676.

(9) Shinar, J. Optically detected magnetic resonance studies of luminescence-quenching processes in pi-conjugated materials and organic light-emitting devices. Laser Phot. Rev. 2012, 6 (6), 767-786.

(10) Wrachtrup, J.; von Borczyskowski, C.; Bernard, J.; Orrit, M.; Brown, R. Optical detection of magnetic resonance in a single molecule. Nature 1993, 363 (6426), 244-245.

(11) Köhler, J.; Disselhorst, J.; Donckers, M.; Groenen, E. J. J.; Schmidt, J.; Moerner, W. E. Magnetic resonance of a single molecular spin. Nature 1993, 363 (6426), 242-244.

(12) Zondervan, R.; Kulzer, F.; Orlinskii, S. B.; Orrit, M. Photoblinking of rhodamine $6 \mathrm{G}$ in poly(vinyl alcohol): Radical dark state formed through the triplet. J. Phys. Chem. A 2003, 107 (35), 6770-6776.

(13) Brouwer, A. C. J.; Groenen, E. J. J.; Schmidt, J. Detecting magnetic resonance through quantum jumps of single molecules. Phys. Rev. Lett. 1998, 80 (18), 3944-3947.

(14) Basché, T.; Kummer, S.; Bräuchle, C. Direct spectroscopic observation of quantum jumps of a single molecule. Nature 1995, 373 (6510), 132-134.

(15) Kulzer, F.; Kummer, S.; Basché, T.; Bräuchle, C. Quantum jumps of single molecules: A method to measure triplet kinetics. J. Inf. Rec. 1996, 22 (5-6), 567-572.

(16) Ha, T.; Enderle, T.; Chemla, D. S.; Selvin, P. R.; Weiss, S. Quantum jumps of single molecules at room temperature. Chem. Phys. Lett. 1997, 271 (1-3), 1-5.

(17) Hofkens, J.; Cotlet, M.; Vosch, T.; Tinnefeld, P.; Weston, K. D.; Ego, C.; Grimsdale, A.; Müllen, K.; Beljonne, D.; Brédas, J. L.; et al. Revealing competitive Förster-type resonance energy-transfer pathways in single bichromophoric molecules. Proc. Natl. Acad. Sci. U. S. A. 2003, 100, 13146-13151.

(18) Yu, J.; Lammi, R.; Gesquiere, A. J.; Barbara, P. F. Singlettriplet and triplet-triplet interactions in conjugated polymer single molecules. J. Phys. Chem. B 2005, 109 (20), 10025-10034.

(19) Orrit, M. Photon statistics in single molecule experiments. Single Mol. 2002, 3 (5-6), 255-265.
(20) Steiner, F.; Vogelsang, J.; Lupton, J. M. Singlet-triplet annihilation limits exciton yield in Poly(3-Hexylthiophene). Phys. Rev. Lett. 2014, 112 (13), 137402.

(21) Veerman, J. A.; Garcia-Parajo, M. F.; Kuipers, L.; van Hulst, N. F. Time-varying triplet state lifetimes of single molecules. Phys. Rev. Lett. 1999, 83 (11), 2155-2158.

(22) Würsch, D.; Hofmann, F. J.; Eder, T.; Aggarwal, A. V.; Idelson, A.; Hoeger, S.; Lupton, J. M.; Vogelsang, J. Molecular Water Lilies: Orienting single molecules in a polymer film by solvent vapor annealing. J. Phys. Chem. Lett. 2016, 7 (22), 4451-4457.

(23) Hübner, C. G.; Renn, A.; Renge, I.; Wild, U. P. Direct observation of the triplet lifetime quenching of single dye molecules by molecular oxygen. J. Chem. Phys. 2001, 115 (21), 9619-9622.

(24) Hofkens, J.; Schroeyers, W.; Loos, D.; Cotlet, M.; Köhn, F.; Vosch, T.; Maus, M.; Herrmann, A.; Müllen, K.; Gensch, T.; De Schryver, F. C. Triplet states as non-radiative traps in multichromophoric entities: single molecule spectroscopy of an artificial and natural antenna system. Spectrochim. Acta, Part A 2001, 57 (11), 2093-2107.

(25) Steiner, F.; Lupton, J. M.; Vogelsang, J. Role of triplet-state shelving in organic photovoltaics: Single-chain aggregates of Poly(3hexylthiophene) versus mesoscopic multichain aggregates. J. Am. Chem. Soc. 2017, 139 (29), 9787-9790.

(26) Yost, S. R.; Hontz, E.; Yeganeh, S.; Van Voorhis, T. Triplet vs singlet energy transfer in organic semiconductors: The tortoise and the hare. J. Phys. Chem. C 2012, 116 (33), 17369-17377.

(27) Schedlbauer, J.; Wilhelm, P.; Grabenhorst, L.; Federl, M. E.; Lalkens, B.; Hinderer, F.; Scherf, U.; Höger, S.; Tinnefeld, P.; Bange, S.; et al. Ultrafast single-molecule fluorescence measured by femtosecond double-pulse excitation photon antibunching. Nano Lett. 2020, 20 (2), 1074-1079.

(28) Weston, K. D.; Dyck, M.; Tinnefeld, P.; Müller, C.; Herten, D. P.; Sauer, M. Measuring the number of independent emitters in single-molecule fluorescence images and trajectories using coincident photons. Anal. Chem. 2002, 74 (20), 5342-5349.

(29) Yip, W. T.; Hu, D.; Yu, J.; Vanden Bout, D. A.; Barbara, P. F. Classifying the photophysical dynamics of single- and multiplechromophoric molecules by single molecule spectroscopy. J. Phys. Chem. A 1998, 102, 7564-7575.

(30) Haase, M.; Hübner, C. G.; Nolde, F.; Müllen, K.; Basché, T. Photoblinking and photobleaching of rylene diimide dyes. Phys. Chem. Chem. Phys. 2011, 13 (5), 1776-1785.

(31) Hania, P. R.; Thomsson, D.; Scheblykin, I. G. Host matrix dependent fluorescence intensity modulation by an electric field in single conjugated polymer chains. J. Phys. Chem. B 2006, 110 (51), 25895-25900.

(32) Schindler, F.; Lupton, J. M.; Feldmann, J. Spontaneous switching of permanent dipoles in single conjugated polymer molecules. Chem. Phys. Lett. 2006, 428 (4-6), 405-410.

(33) Schindler, F.; Lupton, J. M.; Müller, J.; Feldmann, J.; Scherf, U. How single conjugated polymer molecules respond to electric fields. Nat. Mater. 2006, 5 (2), 141-146.

(34) van Eersel, H.; Bobbert, P. A.; Janssen, R. A. J.; Coehoorn, R. Effect of Förster-mediated triplet-polaron quenching and triplet-triplet annihilation on the efficiency roll-off of organic light-emitting diodes. J. Appl. Phys. 2016, 119 (16), 163102.

(35) Reufer, M.; Lagoudakis, P. G.; Walter, M. J.; Lupton, J. M.; Feldmann, J.; Scherf, U. Evidence for temperature-independent triplet diffusion in a ladder-type conjugated polymer. Phys. Rev. B: Condens. Matter Mater. Phys. 2006, 74 (24), 241201.

(36) Schindler, F.; Jacob, J.; Grimsdale, A. C.; Scherf, U.; Müllen, K.; Lupton, J. M.; Feldmann, J. Counting chromophores in conjugated polymers. Angew. Chem., Int. Ed. 2005, 44 (10), 1520-1525.

(37) Hollars, C. W.; Lane, S. M.; Huser, T. Controlled non-classical photon emission from single conjugated polymer molecules. Chem. Phys. Lett. 2003, 370 (3-4), 393-398.

(38) Kumar, P.; Lee, T. H.; Mehta, A.; Sumpter, B. G.; Dickson, R. M.; Barnes, M. D. Photon antibunching from oriented semi- 
conducting polymer nanostructures. J. Am. Chem. Soc. 2004, 126 (11), 3376-3377.

(39) Adachi, T.; Vogelsang, J.; Lupton, J. M. Chromophore bending controls fluorescence lifetime in single conjugated polymer chains. $J$. Phys. Chem. Lett. 2014, 5 (12), 2165-2170.

(40) Stangl, T.; Wilhelm, P.; Remmerssen, K.; Höger, S.; Vogelsang, J.; Lupton, J. M. Mesoscopic quantum emitters from deterministic aggregates of conjugated polymers. Proc. Natl. Acad. Sci. U. S. A. 2015, 112 (41), E5560-E5566.

(41) Eder, T.; Stangl, T.; Gmelch, M.; Remmerssen, K.; Laux, D.; Höger, S.; Lupton, J. M.; Vogelsang, J. Switching between H- and Jtype electronic coupling in single conjugated polymer aggregates. Nat. Commun. 2017, 8, 1641.

(42) Datko, B. D.; Grey, J. K. Population dynamics of multiple triplet excitons revealed from time-dependent fluorescence quenching of single conjugated polymer chains. Sci. Rep. 2019, 9, 817.

(43) Datko, B. D.; Grimm, R.; Walwark, D. J.; Burnside, B.; Grey, J. $\mathrm{K}$. Resolving population dynamics and interactions of multiple triplet excitons one molecule at a time. J. Chem. Phys. 2019, 151 (4), 044203. 